\title{
Weighted approval voting
}

Citation for published version (APA):

Massó, J., \& Vorsatz, M. (2006). Weighted approval voting. METEOR, Maastricht University School of Business and Economics. METEOR Research Memorandum No. 038

https://doi.org/10.26481/umamet.2006038

Document status and date:

Published: 01/01/2006

DOI:

10.26481/umamet.2006038

Document Version:

Publisher's PDF, also known as Version of record

\section{Please check the document version of this publication:}

- A submitted manuscript is the version of the article upon submission and before peer-review. There can be important differences between the submitted version and the official published version of record.

People interested in the research are advised to contact the author for the final version of the publication, or visit the DOI to the publisher's website.

- The final author version and the galley proof are versions of the publication after peer review.

- The final published version features the final layout of the paper including the volume, issue and page numbers.

Link to publication

\footnotetext{
General rights rights.

- You may freely distribute the URL identifying the publication in the public portal. please follow below link for the End User Agreement:

www.umlib.nl/taverne-license

Take down policy

If you believe that this document breaches copyright please contact us at:

repository@maastrichtuniversity.nl

providing details and we will investigate your claim.
}

Copyright and moral rights for the publications made accessible in the public portal are retained by the authors and/or other copyright owners and it is a condition of accessing publications that users recognise and abide by the legal requirements associated with these

- Users may download and print one copy of any publication from the public portal for the purpose of private study or research.

- You may not further distribute the material or use it for any profit-making activity or commercial gain

If the publication is distributed under the terms of Article $25 \mathrm{fa}$ of the Dutch Copyright Act, indicated by the "Taverne" license above, 
Jordi Massó, Marc Vorsatz

Weighted Approval Voting

$\mathrm{RM} / 06 / 038$

JEL code: D71

\section{METE@R}

Maastricht research school of Economics of TEchnology and ORganizations

Universiteit Maastricht

Faculty of Economics and Business Administration P.O. Box 616

NL - 6200 MD Maastricht

phone : ++31433883830

fax : ++31433884873 



\title{
Weighted Approval Voting*
}

\author{
Jordi Massó ${ }^{\dagger}$ and Marc Vorsatz
}

September 4, 2006

\begin{abstract}
To allow society to treat unequal alternatives distinctly we propose a natural extension of Approval Voting [7] by relaxing the assumption of neutrality. According to this extension, every alternative receives ex-ante a non-negative and finite weight. These weights may differ across alternatives. Given the voting decisions of every individual (individuals are allowed to vote for, or approve of, as many alternatives as they wish to), society elects all alternatives for which the product of total number of votes times exogenous weight is maximal. Our main result is an axiomatic characterization of this voting procedure.
\end{abstract}

Keywords: Approval Voting, Neutrality.

JEL-Number: D71.

\section{Introduction}

Approval Voting [7] is perhaps the most well known voting procedure that has been proposed as an alternative to the Plurality Rule. For example, in a recent survey Brams and Fishburn [8] lay out that the United Nations General Assembly, several scientific institutions (among others the Mathematical Association of America, the American Mathematical

*We thank Salvador Barberà for his helpful comments at early stages of this research. We also thank Miguel Ángel Ballester, Steven Brams, Antonio Nicolò, Pedro Rey, Arunava Sen, John Weymark, and the participants of the UAB Lunch Seminar on Game Theory and Social Choice for their useful comments and suggestions. The work of Jordi Massó is partially supported by the Spanish Ministerio de Educación y Ciencia through grant SEJ2005-04081 and by the Generalitat de Catalunya, through the Barcelona Economics Program (CREA) and the grant SGR2005-00454.

†Departament d'Economia i d'Història Econòmica and CODE. Universitat Autònoma de Barcelona, 08193 Bellaterra (Barcelona), Spain. E-mail: jordi.masso@uab.es

${ }^{\ddagger}$ Corresponding author. Department of Economics, Maastricht University, P.O. Box 616, 6200 MD Maastricht, The Netherlands. E-mail: m.vorsatz@algec.unimaas.nl 
Society, the American Statistical Association), and some political parties (i.e., in Pennsylvania) adopted Approval Voting or had at least some experience with it. According to this rule, every voter can vote for, or approve of, as many alternatives as s/he wishes to and given the response profile of individual approvals, society elects the set of alternatives with the highest amount of total votes.

Very recently, it has been looked at Approval Voting from different points of view. First, several case studies have been carried out. For instance, Laslier [16] studies the 1999 elections of the President and the Council of the Society for Social Choice and Welfare, where Approval Voting was the method being used but voters were also asked to submit their rankings under the Borda count. Laslier [17] analyzes Approval Voting by means of an experiment carried out in six places in France during the first round of the presidential election of 2002, in which Jean-Marie Le Pen came in second, defeating the socialist candidate Lionel Jospin, and thus, obtained the right to compete in the second round against Jacques Chirac. Second, wide theoretical research has also been under way. Regenwetter and Tsetlin [19] compare Approval Voting with positional voting methods and identify conditions under which they tend to agree. Vorsatz [23] shows that, on the domain of dichotomous preferences, Approval Voting coincides with the Borda count. De Sinopoli et al. [10] analyze strategic behavior in Approval Voting games. Brams and Sanver [9] study Approval Voting under the assumption that voters do not only have preferences on the set of alternatives but also judgements about their acceptability. Nitzan and Baharad [18] study the consequence of modifying Approval Voting by restricting the minimal and maximal number of alternatives that can be voted for, and, finally, Dellis and Oak [11] compare Approval Voting with the Plurality Rule in a political competition model with endogenous candidacy entry.

It is inherent in the definition of Approval Voting that every vote counts the same, independently which alternative receives it. We believe that this neutrality assumption is relevant in democratic processes where all alternatives should be treated equally (i.e., presidential elections), but may not be as natural for group decision making problems in which the characteristics of the alternatives are objectively different and society agrees on 
the desirability to treat unequal alternatives distinctly (i.e., in the case when alternatives are candidates seniority, age, education, race, gender, and others may matter). Hence, it is our objective to propose a natural extension of Approval Voting and present an axiomatic characterization of this new voting procedure.

The generalization we consider, Weighted Approval Voting, is simple and intuitive at the same time: Assign ex-ante to every alternative a non-negative and finite weight. Observe that the weights are potentially different for distinct alternatives. Given the approvals of every voter (again voters can vote for as many alternatives as they wish to), society elects the set of alternatives for which the product of total number of votes times weight is maximal. This voting rule reduces to Approval Voting when the weights are identical and strictly positive for all alternatives.

We are interested in general voting procedures that could operate in different voting situations in which the set of voters as well as the set of alternatives might vary (for instance, different choices have to be made over time). In particular, and given a universal set of potential voters and a set of conceivable alternatives, a voting procedure (a family of voting rules) should specify an outcome for every electorate (the subset of voters that indeed vote) and every set of feasible alternatives (the subset of alternatives that are indeed at stake). Thus, our axiomatic analysis of Weighted Approval Voting allows for a variable electorate and a variable set of feasible alternatives. Consequently, we require the satisfaction of two consistency conditions that link the elected outcome made by the voting procedure at different situations; namely, they keep track on how the set of elected alternatives changes as the electorate or the set of feasible alternatives varies. Consistency in alternatives requires that if the set of feasible alternatives is reduced, then the set of elected alternatives in the new situation coincides with the set of elected alternatives in the original situation restricted to the new set of feasible alternatives (provided that this set is not empty). This is an Independence of Irrelevant Alternatives like condition that conveys a separability feature to the voting procedure: a general election from a set of feasible alternatives can be reduced to choices among pairs of alternatives only. Consistency in alternatives insures then that collecting the choices made at each of the pairs coincides with 
the general election. In our case, consistency of alternatives will guarantee additionally the transitivity of the weights and hence, it will be possible to focus mainly on elections from sets with only two alternatives. These are the reasons why we can define the other four properties only with respect to two alternatives, say $x$ and $y$. Consistency in voters asks that if two disjoint electorates elect from the set $\{x, y\}$ a common set of alternatives, then exactly this set has to be elected whenever all voters within the two electorates and no voter outside the two electorates participates in the election. According to the third property, anonymity, the only information taken into account is how many votes $x$ and $y$ receive (and not from whom the votes come). The no-support condition states that whenever $x$ does not receive any vote, then $y$ has to be elected for sure (observe that it is still possible to elect both $x$ and $y$ and that, like Approval Voting does, at least one alternative has to be elected). Finally, coherence requires that if $x$ is the unique elected alternative in an electorate that only votes for $x$, then there is some other response profile (with strictly positive support for both alternatives, $x$ and $y$ ) at which $x$ is elected (perhaps together with $y$ ). In particular, coherence eliminates constant-like voting rules according to which one alternative is the unique elected alternative on the domain where both alternatives receive at least one vote.

Our result states that a voting procedure is consistent in alternatives and voters, anonymous, coherent, and satisfies the non-support condition if and only if it is a Weighted Approval Voting.

Several authors have analyzed Approval Voting axiomatically. Fishburn [14] shows that if the set of alternatives is fixed and the electorate is allowed to vary, then Approval Voting is characterized by means of consistency in voters, neutrality, anonymity and disjoint equality (if two voters approve two nonempty and disjoint subsets of feasible alternatives, then the union of these two sets has to be elected when there is no other voter). Fishburn [13] also characterizes Approval Voting by means of consistency in voters, neutrality, faithfulness (if there is only one voter and this voter approves at least one alternative, the voting procedure selects all alternatives this voter supports), and cancelation (if all alternative receive the same number of votes, all alternatives are elected). Alós-Ferrer [2] shows that 
this last characterization is not tight since neutrality follows from consistency in voters, faithfulness, and cancelation. Sertel [20] presents an alternative definition of Approval Voting that differs from the original one only in the situation when no alternative receives any vote. He assumes in this case that no alternative is elected. This, slightly different, voting rule is then characterized by anonymity, weak unanimity (if the society consists of only one voter, the voting procedure selects the alternatives that the voter supports), weak consistency (this property weakens consistency in voters slightly without changing its main idea), and strong disjoint equality (disjoint equality is also defined for the case when some voter does not approve any alternative). A further characterization is due to Baigent and $\mathrm{Xu}$ [4]. They apply the properties of neutrality, strict monotonicity (if $x$ is elected at a certain response profile and a second response profile is identical to the first one apart from the fact that $x$ receives now an additional vote, then only $x$ is elected at the second response profile) and independence of symmetric substitutions. The latter condition requires that the set of elected alternatives should be the same in the following two situations: In the first situation, some voter approves, among other alternatives, $x$ but not $y$, whereas another voter approves, among other alternatives, $y$ but not $x$. The second situation is identical to the first one with the only difference that the first voter approves now $y$ but not $x$ and the second voter approves now $x$ but not $y$. Goodin and List [15] relate Approval Voting axiomatically to May's Theorem by showing that Approval Voting is characterized by anonymity (they define anonymity in a different way than we do, yet, the two properties turn out to be equivalent), neutrality, and strict monotonicity. Finally, Fishburn [13] and Vorsatz [22] study Approval Voting axiomatically under the assumption that individuals have dichotomous preferences (voters divide alternatives into two sets, the set of good alternatives and the set of bad alternatives; see, for instance, [5]), the context in which Approval Voting and its extensions become social choice functions. While the first paper concentrates on the notion of strategy-proofness (incentives to represent preferences truthfully), the latter explores additionally the efficiency of Approval Voting. Observe that any Weighted Approval Voting (with strictly positive weights) satisfies strategy-proofness but only Approval Voting is efficient whenever voters have dichotomous preferences. In fact, 
if the weights are derived from some equity principle, one introduces a trade-off between efficiency and equity because more unequal weights lead to a less efficient outcome but stress the underlying equity principle more. Additionally, any Weighted Approval Voting with strictly positive weights satisfies strict monotonicity and independence of symmetric substitutions while it fails to satisfy axioms that have neutrality inherent to its definition as disjoint equality, faithfulness, cancelation, and weak unanimity.

The remainder of the paper is organized as follows. In the next Section, we introduce our notation and main definitions. In Section 3, we present the five axioms that characterize Weighted Approval Voting. Afterwards, we prove our theorem. Finally, we establish the independence of the axioms and conclude with some remarks.

\section{Preliminaries}

We consider elections in which the set of alternatives and the set of voters may vary. First, let $\mathcal{K}$ be the universal set of conceivable alternatives for election. Generic alternatives will be denoted by $x, y$ and $z$. The cardinality of $\mathcal{K}, \kappa$, is finite and greater or equal to 3 (if the set of universal alternatives contains only two alternatives, then the first axiom, consistency in alternatives, is superfluous in Theorem 1 as it will become clear later on). Since it may happen that not all conceivable alternatives are eligible, we restrict the set of feasible alternatives to be equal to $K \subseteq \mathcal{K}$. Alternatively, we will denote subsets of alternatives by the capital letters $S$ or $T$. Second, we represent the universal set of voters by the set of natural numbers $\mathbb{N}$. We will consider situations in which the set of voters actually participating in the election, the electorate $N$, is a finite subset of the natural numbers. Often we will also use the capital letters $A$ and $B$ to denote electorates. The cardinalities of $N$ and $K$ are equal to $n \geq 1$ and $k \geq 2$, respectively.

For any voter $i \in \mathbb{N}$, let $M_{i} \in 2^{\mathcal{K}}$ be the set of alternatives $i$ votes for. A profile $M=\left(M_{i}\right)_{i \in \mathbb{N}} \in\left(2^{\mathcal{K}}\right)^{\mathbb{N}}$ is a list of all votes. Given a profile $M$ and an electorate $N$, a response profile $M_{N}=\left(M_{i}\right)_{i \in N} \in\left(2^{\mathcal{K}}\right)^{N}$ is the $n$-tuple of votes coming from the electorate $N$ at profile $M$. We say that the response profiles $M_{A}$ and $M_{B}$, corresponding to the electorates 
$A$ and $B$ of equal size, are isomorphic if there exists a one-to-one mapping $\pi: A \rightarrow B$ such that for all $i \in A, M_{i}=M_{\pi(i)}$. Given two disjoint electorates $A$ and $B$ and two response profiles $M_{A}$ and $M_{B}$, denote by $M_{A}+M_{B}$ the response profile $\left(M_{i}\right)_{i \in A \cup B} \in\left(2^{\mathcal{K}}\right)^{A \cup B}$. Given the response profile $M_{N}$ and alternative $x \in \mathcal{K}$, let $G_{x}\left(M_{N}\right)=\left|\left\{i \in N: x \in M_{i}\right\}\right|$ be the support of $x$ at $M_{N}$.

Given a set of feasible alternatives $K$ and an electorate $N$, a voting rule $v^{K, N}:\left(2^{\mathcal{K}}\right)^{\mathbb{N}} \rightarrow$ $2^{K} \backslash\{\emptyset\}$ selects, for all profiles $M$, a nonempty set of feasible alternatives $v^{K, N}(M)$ with the property that for all $M, M^{\prime} \in\left(2^{\mathcal{K}}\right)^{\mathbb{N}}$ such that $M_{N}=M_{N}^{\prime}, v^{K, N}(M)=v^{K, N}\left(M^{\prime}\right)$. This is the reason why, with a slight abuse of notation, we will write $v^{K}\left(M_{N}\right)$ instead of $v^{K, N}(M)$. Observe first that, although the empty set can be a component of response profiles, the images of a given voting rule are nonempty subsets of feasible alternatives. We exclude the possibility to elect no alternative (even when all feasible alternatives get zero support), because we want to include the interpretation of the image as the set of pre-selected alternatives from which an ultimate winning alternative has still to be determined in a yet to be specified way (i.e., a lottery). Additionally, we aim at generalizing Approval Voting which, for each response profile, elects the (always nonempty) subset of feasible alternatives with maximal support. Second, response profiles may include votes for unfeasible alternatives. These votes are redundant but this formulation simplifies later on the definition of consistency in alternatives.

A family of voting rules $\left\{v^{K, N}:\left(2^{\mathcal{K}}\right)^{\mathbb{N}} \rightarrow 2^{K} \backslash\{\emptyset\}\right\}_{K, N}$ is a set of voting rules, one for every set of feasible alternatives $K$ and electorate $N$. It is denoted by $v$. Given the family of voting rules $v$ and a particular set of feasible alternatives $K$, we denote the subfamily of voting rules $\left\{v^{K, N}:\left(2^{\mathcal{K}}\right)^{\mathbb{N}} \rightarrow 2^{K} \backslash\{\emptyset\}\right\}_{N}$ by $v^{K}$.

As we have already argued in the Introduction, there are meaningful situations in which not all alternatives are equally important. Thus, it is our objective to eliminate the neutrality assumption underlying Approval Voting by allowing for the possibility to discriminate among alternatives keeping the impact of a vote for a given alternative the same for all voters. ${ }^{1}$ For instance, in some elections alternatives may be candidates with

\footnotetext{
${ }^{1}$ An alternative approach aims at allowing for different weights for distinct voters maintaining neutrality.
} 
different characteristics like seniority, sex, age, degree of qualification, field of specialization, etc. In these elections society may not find it desirable to treat all candidates alike. To define the natural non-neutral extension of Approval Voting, let $\mathbb{R}_{+}$be the set of nonnegative real numbers and let $\mathbb{R}_{++}$be the set of strictly positive real numbers.

Definition 1 The family of voting rules $v$ is a Weighted Approval Voting if there exists a vector of weights $p=\left(p_{x}\right)_{x \in \mathcal{K}}$, with $p_{x} \in \mathbb{R}_{+}$for all $x \in \mathcal{K}$, such that for all sets of feasible alternatives $K$, all profiles $M \in\left(2^{\mathcal{K}}\right)^{\mathbb{N}}$, and all electorates $N$,

$$
x \in v^{K}\left(M_{N}\right) \text { if and only if } p_{x} \cdot G_{x}\left(M_{N}\right) \geq p_{y} \cdot G_{y}\left(M_{N}\right) \text { for all } y \in K .
$$

The family of Weighted Approval Voting with the vector of weights $p$ is denoted by $v_{p}$. Approval Voting, denoted $v_{A}$, is the special case of a Weighted Approval Voting when for all

$x, y \in \mathcal{K}, p_{x}=p_{y} \in \mathbb{R}_{++}$. Observe first that the constant rule, which elects always the set of feasible alternatives, is a Weighted Approval Voting with weight zero for all alternatives. Second, the vector of weights $\left(p_{x}\right)_{x \in \mathcal{K}}$ has one degree of freedom because multiplying the weights by a strictly positive number does not have any effect on the result of the election.

Remark 1 For all weights $p$ and all $\lambda \in \mathbb{R}_{++}, v_{\lambda \cdot p}=v_{p}$.

Finally, given the Weighted Approval Voting $v_{p}$ and the set of feasible alternatives $K$, the subfamily $v_{p}^{K}=\left\{v_{p}^{K, N}:\left(2^{\mathcal{K}}\right)^{\mathbb{N}} \rightarrow 2^{K} \backslash\{\emptyset\}\right\}_{N}$ will be called the Weighted Approval Voting relative to $p^{K}=\left(p_{x}\right)_{x \in K}$.

\section{Properties and Characterization}

We present now formally the properties that characterize all Weighted Approval Voting. Two consistency properties describe how the elected set of alternatives varies as the set of feasible alternatives or the electorate changes.

To analyze the normative foundations of this generalization of Approval Voting is also very prospective because one can identify a variety situations where this rule is applied. Examples include voting in the EU Member Council (the weight of a country is determined by its population size) and management boards (a vote from the CEO counts usually more than a weight from other board members). To our best knowledge, this rule has not been studied axiomatically so far. 
Consistency in Alternatives: The family of voting rules $v$ is consistent in alternatives if for all sets of feasible alternatives $S \subset T \subseteq \mathcal{K}$, all profiles $M \in\left(2^{\mathcal{K}}\right)^{\mathbb{N}}$, and all electorates $N$ such that $v^{T}\left(M_{N}\right) \cap S \neq \emptyset$,

$$
v^{S}\left(M_{N}\right)=v^{T}\left(M_{N}\right) \cap S
$$

This property means the following. Assume first that a particular set of alternatives is feasible and society elects a subset of them. If it turns out afterwards that fewer alternatives are feasible, then the set of elected alternatives is restricted accordingly (see [3]). Consistency in alternatives plays a crucial role in the proof of our characterization because it establishes the transitivity of the weights and allows us to extend the two alternatives case to any set of alternatives. For the latter reason we only have to state the other four properties with respect to two alternatives.

The second consistency property requires that if two disjoint electorates elect some common alternatives, then exactly these alternatives are elected when the two electorates are assembled (see [21]). This property insures the additivity of the votes.

CONSISTEnCy In Voters: The family of voting rules $v$ is consistent in voters if for all alternatives $x, y \in \mathcal{K}$, all profiles $M \in\left(2^{\mathcal{K}}\right)^{\mathbb{N}}$, and all disjoint electorates $A, B \subseteq \mathbb{N}$ such that $v^{\{x, y\}}\left(M_{A}\right) \cap v^{\{x, y\}}\left(M_{B}\right) \neq \emptyset$,

$$
v^{\{x, y\}}\left(M_{A}+M_{B}\right)=v^{\{x, y\}}\left(M_{A}\right) \cap v^{\{x, y\}}\left(M_{B}\right) .
$$

According to the third property the set of elected alternatives depends only on the support of the alternatives (and hence, the weights will be independent of the identity of the voters). Formally,

AnONymity: The family of voting rules $v$ is anonymous if for all alternatives $x, y \in \mathcal{K}$, all profiles $M, M^{\prime} \in\left(2^{\mathcal{K}}\right)^{\mathbb{N}}$, and all electorates $A$ and $B$ such that $G_{x}\left(M_{A}\right)=G_{x}\left(M_{B}^{\prime}\right)$ and $G_{y}\left(M_{A}\right)=G_{y}\left(M_{B}^{\prime}\right)$,

$$
v^{\{x, y\}}\left(M_{A}\right)=v^{\{x, y\}}\left(M_{B}^{\prime}\right)
$$

The fourth axiom refers to response profiles with the property that the support of at least one of the two feasible alternatives is zero. 
No-SupporT: The family of voting rules $v$ satisfies the no-support condition if for all alternatives $x, y \in \mathcal{K}$, all profiles $M \in\left(2^{\mathcal{K}}\right)^{\mathbb{N}}$, and all electorates $N$ such that $G_{x}\left(M_{N}\right)=0$,

$$
y \in v^{\{x, y\}}\left(M_{N}\right) \text {. }
$$

The last property, coherence, requires basically that for both alternatives $x$ and $y$ there is some electorate and some response profile (with strictly positive support for both alternatives) such that the considered alternative is elected.

CoHerence: The family of voting rules $v$ is coherent if for all alternatives $x, y \in \mathcal{K}$, all profiles $M \in\left(2^{\mathcal{K}}\right)^{\mathbb{N}}$, and all electorates $A$ such that $G_{x}\left(M_{A}\right)>0$ and $G_{y}\left(M_{A}\right)=0$, if $v^{\{x, y\}}\left(M_{A}\right)=\{x\}$ then there exist a profile $M^{\prime} \in\left(2^{\mathcal{K}}\right)^{\mathbb{N}}$ and an electorate $N$ with $G_{x}\left(M_{N}^{\prime}\right)>0$ and $G_{y}\left(M_{N}^{\prime}\right)>0$, such that

$$
x \in v^{\{x, y\}}\left(M_{N}^{\prime}\right) .
$$

In Theorem 1 we state an axiomatic characterization of all Weighted Approval Voting based on these five properties.

Theorem 1 The family of voting rules $v$ is consistent in alternatives and voters, anonymous, coherent and satisfies the no-support condition if and only if $v$ is a Weighted Approval Voting.

\section{Proof of the Characterization}

We start by proving that in the case of two feasible alternatives the only relevant information is not the support of the alternatives (as it follows directly from anonymity) but rather the fractions of the supports. Afterwards, we prove a monotonicity like property.

Lemma 1 Assume that the family of voting rules $v$ is consistent in voters and anonymous. Then, for all alternatives $x, y \in \mathcal{K}$, all profiles $M, M^{\prime} \in\left(2^{\mathcal{K}}\right)^{\mathbb{N}}$, and all electorates $A$ and $B$ such that $G_{y}\left(M_{A}\right)>0, G_{y}\left(M_{B}^{\prime}\right)>0$, and $\frac{G_{x}\left(M_{A}\right)}{G_{y}\left(M_{A}\right)}=\frac{G_{x}\left(M_{B}^{\prime}\right)}{G_{y}\left(M_{B}^{\prime}\right)}$,

$$
v^{\{x, y\}}\left(M_{A}\right)=v^{\{x, y\}}\left(M_{B}^{\prime}\right) .
$$


Proof: Let $\{x, y\}$ be the set of feasible alternatives and take any two response profiles $M_{A}$ and $M_{B}^{\prime}$ that satisfy the hypothesis of Lemma 1 . Consider two electorates $\bar{A}$ and $\bar{B}$ of sizes $|A| \cdot G_{y}\left(M_{B}^{\prime}\right)$ and $|B| \cdot G_{y}\left(M_{A}\right)$, respectively. Let $\bar{M}_{\bar{A}}$ and $\bar{M}_{\bar{B}}^{\prime}$ be two response profiles obtained by replicating $G_{y}\left(M_{B}^{\prime}\right)$-times the response profile $M_{A}$ and $G_{y}\left(M_{A}\right)$-times the response profile $M_{B}^{\prime}$, respectively. Namely, the response profile $\bar{M}_{\bar{A}}$ is the union of $G_{y}\left(M_{B}^{\prime}\right)$-isomorphic copies of $M_{A}$ (denoted by $\left.M_{A_{1}}, \ldots, M_{A_{G_{y}\left(M_{B}^{\prime}\right)}}\right)$ and the response profile $\bar{M}_{\bar{B}}^{\prime}$ is the union of $G_{y}\left(M_{A}\right)$-isomorphic copies of $M_{B}^{\prime}$ (denoted by $M_{B_{1}}^{\prime}, \ldots, M_{B_{G_{y}\left(M_{A}\right)}^{\prime}}^{\prime}$ ), where all electorates $A_{t}, t=1, \ldots, G_{y}\left(M_{B}^{\prime}\right)$ and all $B_{r}, r=1, \ldots, G_{x}\left(M_{A}\right)$ are disjoint. Observe that $G_{x}\left(\bar{M}_{\bar{A}}\right)=G_{x}\left(M_{A}\right) \cdot G_{y}\left(M_{B}^{\prime}\right)$ and $G_{x}\left(\bar{M}_{\bar{B}}^{\prime}\right)=G_{x}\left(M_{B}^{\prime}\right) \cdot G_{y}\left(M_{A}\right)$. By assumption,

$$
G_{x}\left(\bar{M}_{\bar{A}}\right)=G_{x}\left(\bar{M}_{\bar{B}}^{\prime}\right) .
$$

Moreover, $G_{y}\left(\bar{M}_{\bar{A}}\right)=G_{y}\left(M_{A}\right) \cdot G_{y}\left(M_{B}^{\prime}\right)$ and $G_{y}\left(\bar{M}_{\bar{B}}^{\prime}\right)=G_{y}\left(M_{B}^{\prime}\right) \cdot G_{y}\left(M_{A}\right)$. Thus,

$$
G_{y}\left(\bar{M}_{\bar{A}}\right)=G_{y}\left(\bar{M}_{\bar{B}}^{\prime}\right) .
$$

By anonymity, (2) and (3) imply

$$
v^{\{x, y\}}\left(\bar{M}_{\bar{A}}\right)=v^{\{x, y\}}\left(\bar{M}_{\bar{B}}^{\prime}\right) .
$$

Also, by anonymity, for all $t=1, \ldots, G_{y}\left(M_{B}^{\prime}\right)$ and all $r=1, \ldots, G_{y}\left(M_{A}\right), v^{\{x, y\}}\left(M_{A_{t}}\right)=$ $v^{\{x, y\}}\left(M_{A}\right)$ and $v^{\{x, y\}}\left(M_{B_{r}}^{\prime}\right)=v^{\{x, y\}}\left(M_{B}^{\prime}\right)$. Then, by iterating on the properties of consistency in voters and anonymity,

$$
v^{\{x, y\}}\left(\bar{M}_{\bar{A}}\right)=v^{\{x, y\}}\left(\sum_{t=1}^{G_{y}\left(M_{B}^{\prime}\right)} M_{A_{t}}\right)=\bigcap_{t=1}^{G_{y}\left(M_{B}^{\prime}\right)} v^{\{x, y\}}\left(M_{A_{t}}\right)=v^{\{x, y\}}\left(M_{A}\right)
$$

and

$$
v^{\{x, y\}}\left(\bar{M}_{\bar{B}}^{\prime}\right)=v^{\{x, y\}}\left(\sum_{r=1}^{G_{y}\left(M_{A}\right)} M_{B_{r}}^{\prime}\right)=\bigcap_{r=1}^{G_{y}\left(M_{A}\right)} v^{\{x, y\}}\left(M_{B_{r}}^{\prime}\right)=v^{\{x, y\}}\left(M_{B}^{\prime}\right) .
$$

By (4), $v^{\{x, y\}}\left(M_{A}\right)=v^{\{x, y\}}\left(M_{B}^{\prime}\right)$.

Lemma 2 Assume that the family of voting rules $v$ is consistent in voters, anonymous, and satisfies the no-support condition. Then, for all alternatives $x, y \in \mathcal{K}$, all profiles $M, M^{\prime} \in$ $\left(2^{\mathcal{K}}\right)^{\mathbb{N}}$, and all electorates $A$ and $B$ such that $G_{y}\left(M_{A}\right)>0, G_{y}\left(M_{B}^{\prime}\right)>0, \frac{G_{x}\left(M_{A}\right)}{G_{y}\left(M_{A}\right)}>\frac{G_{x}\left(M_{B}^{\prime}\right)}{G_{y}\left(M_{B}\right)}$, and $x \in v^{\{x, y\}}\left(M_{B}^{\prime}\right)$,

$$
x \in v^{\{x, y\}}\left(M_{A}\right) .
$$


Proof: Let $\{x, y\}$ be the set of feasible alternatives and take any two response profiles $M_{A}$ and $M_{B}^{\prime}$ that satisfy the hypothesis of Lemma 2. Consider two electorates $\bar{A}$ and $\bar{B}$ of sizes $|A| \cdot G_{y}\left(M_{B}^{\prime}\right)$ and $|B| \cdot G_{y}\left(M_{A}\right)$, respectively. Let $\bar{M}_{\bar{A}}$ and $\bar{M}_{\bar{B}}^{\prime}$ be the two response profiles obtained by replicating $G_{y}\left(M_{B}^{\prime}\right)$-times the response profile $M_{A}$ and $G_{y}\left(M_{A}\right)$-times the response profile $M_{B}^{\prime}$, respectively. Namely, the response profile $\bar{M}_{\bar{A}}$ is the union of $G_{y}\left(M_{B}^{\prime}\right)$-isomorphic copies of $M_{A}$ and the response profile $\bar{M}_{\bar{B}}^{\prime}$ is the union of $G_{y}\left(M_{A}\right)$ isomorphic copies of $M_{B}^{\prime}$. By consistency in voters and anonymity,

$$
v^{\{x, y\}}\left(\bar{M}_{\bar{A}}\right)=v^{\{x, y\}}\left(M_{A}\right) \text { and } v^{\{x, y\}}\left(\bar{M}_{\bar{B}}^{\prime}\right)=v^{\{x, y\}}\left(M_{B}^{\prime}\right) .
$$

Observe that $G_{y}\left(\bar{M}_{\bar{A}}\right)=G_{y}\left(M_{A}\right) \cdot G_{y}\left(M_{B}^{\prime}\right)=G_{y}\left(\bar{M}_{\bar{B}}^{\prime}\right)$. Moreover, by hypothesis, $G_{x}\left(\bar{M}_{\bar{A}}\right)=$ $G_{x}\left(M_{A}\right) \cdot G_{y}\left(M_{B}^{\prime}\right)>G_{y}\left(M_{A}\right) \cdot G_{x}\left(M_{B}^{\prime}\right)=G_{x}\left(\bar{M}_{\bar{B}}^{\prime}\right)$.

Now, take two response profiles $\hat{M}_{C}$ and $\hat{M}_{D}$ corresponding to the disjoint electorates $C$ and $D$, with the properties that $G_{y}\left(\hat{M}_{D}\right)=G_{y}\left(\bar{M}_{\bar{B}}^{\prime}\right), G_{x}\left(\hat{M}_{D}\right)=G_{x}\left(\bar{M}_{\bar{B}}^{\prime}\right), G_{y}\left(\hat{M}_{C}\right)=0$, and $G_{x}\left(\hat{M}_{C}\right)=G_{x}\left(\bar{M}_{\bar{A}}\right)-G_{x}\left(\bar{M}_{\bar{B}}^{\prime}\right)$. By anonymity,

$$
v^{\{x, y\}}\left(\hat{M}_{D}\right)=v^{\{x, y\}}\left(\bar{M}_{\bar{B}}^{\prime}\right) .
$$

Since $G_{x}\left(\bar{M}_{\bar{A}}\right)=G_{x}\left(\hat{M}_{C}\right)+G_{x}\left(\bar{M}_{\bar{B}}^{\prime}\right)=G_{x}\left(\hat{M}_{C}\right)+G_{x}\left(\hat{M}_{D}\right), G_{y}\left(\bar{M}_{\bar{A}}\right)=G_{y}\left(\hat{M}_{C}\right)+G_{y}\left(\hat{M}_{D}\right)$, and the electorates $C$ and $D$ are disjoint, $G_{x}\left(\bar{M}_{\bar{A}}\right)=G_{x}\left(\hat{M}_{C}+\hat{M}_{D}\right)$ and $G_{y}\left(\bar{M}_{\bar{A}}\right)=$ $G_{y}\left(\hat{M}_{C}+\hat{M}_{D}\right)$. By anonymity,

$$
v^{\{x, y\}}\left(\bar{M}_{\bar{A}}\right)=v^{\{x, y\}}\left(\hat{M}_{C}+\hat{M}_{D}\right) .
$$

By the no-support condition,

$$
x \in v^{\{x, y\}}\left(\hat{M}_{C}\right) .
$$

Since, by hypothesis, $x \in v^{\{x, y\}}\left(M_{B}^{\prime}\right)$, conditions (5) and (6) imply $x \in v^{\{x, y\}}\left(\hat{M}_{D}\right)$. Therefore,

$$
x \in v^{\{x, y\}}\left(\hat{M}_{C}\right) \cap v^{\{x, y\}}\left(\hat{M}_{D}\right) \neq \emptyset .
$$

By consistency in voters,

$$
v^{\{x, y\}}\left(\hat{M}_{C}+\hat{M}_{D}\right)=v^{\{x, y\}}\left(\hat{M}_{C}\right) \cap v^{\{x, y\}}\left(\hat{M}_{D}\right) .
$$

Conditions (7), (8), and (9) imply that $x \in v^{\{x, y\}}\left(\bar{M}_{\bar{A}}\right)$. Finally, it follows from (5) that $x \in v^{\{x, y\}}\left(M_{A}\right)$. 
Given a family of voting rules $v$ we will now construct, for every set $\{x, y\}$ of feasible alternatives, two weights, $p_{x}^{\{x, y\}} \in \mathbb{R}_{+}$and $p_{y}^{\{x, y\}} \in \mathbb{R}_{+}$, for which the subfamily of voting rules $v^{\{x, y\}}$ is the Weighted Approval Voting relative to $p^{\{x, y\}}=\left(p_{x}^{\{x, y\}}, p_{y}^{\{x, y\}}\right)$.

Lemma 3 Assume that the family of voting rules $v$ is consistent in voters, anonymous, coherent and satisfies the no-support condition. Then, for all alternatives $x, y \in \mathcal{K}$ there exist two weights $p_{x}^{\{x, y\}}, p_{y}^{\{x, y\}} \in \mathbb{R}_{+}$such that $v^{\{x, y\}}$ is the Weighted Approval Voting relative to $p^{\{x, y\}}=\left(p_{x}^{\{x, y\}}, p_{y}^{\{x, y\}}\right)$.

Proof: Let $\{x, y\}$ be the set of feasible alternatives and take any $v$ that satisfies the hypothesis of Lemma 3. We will show that there exist two weights $p_{x}^{\{x, y\}} \in \mathbb{R}_{+}$and $p_{y}^{\{x, y\}} \in \mathbb{R}_{+}$with the property that for all profiles $M$, all electorates $N$, and all $z \in\{x, y\}$,

$$
z \in v^{\{x, y\}}\left(M_{N}\right) \Leftrightarrow p_{z}^{\{x, y\}} \cdot G_{z}\left(M_{N}\right) \geq p_{w}^{\{x, y\}} \cdot G_{w}\left(M_{N}\right) \text { for all } w \in\{x, y\} .
$$

To insure that condition (10) holds, we investigate the restrictions that response profiles impose on the weights. Consider any electorate $N$ and let the response profile $M_{N}$ be such that $G_{x}\left(M_{N}\right)=0$ and $G_{y}\left(M_{N}\right)=0$. Since $v$ satisfies the no-support condition, $v^{\{x, y\}}\left(M_{N}\right)=\{x, y\}$. Then, given $M_{N}$, condition (10) holds for any $p_{x}^{\{x, y\}} \in \mathbb{R}_{+}$and $p_{y}^{\{x, y\}} \in \mathbb{R}_{+}$. So, for all electorates $N$, response profiles with the property that $G_{x}\left(M_{N}\right)=$ $G_{y}\left(M_{N}\right)=0$ do not impose any restriction on the weights.

In the next step, we focus on two response profiles that give one vote to one alternative and zero votes to the other. Formally, given any electorate $N$, let $M_{N}$ and $M_{N}^{\prime}$ be such that $G_{x}\left(M_{N}\right)=1, G_{y}\left(M_{N}\right)=0, G_{x}\left(M_{N}^{\prime}\right)=0$ and $G_{y}\left(M_{N}^{\prime}\right)=1$. By the no-support condition, $x \in v^{\{x, y\}}\left(M_{N}\right)$ and $y \in v^{\{x, y\}}\left(M_{N}^{\prime}\right)$. The latter two conditions restrict the two images to be $v^{\{x, y\}}\left(M_{N}\right) \in\{\{x\},\{x, y\}\}$ and $v^{\{x, y\}}\left(M_{N}^{\prime}\right) \in\{\{y\},\{x, y\}\}$. We consider the four cases separately.

1. Suppose that $v^{\{x, y\}}\left(M_{N}\right)=v^{\{x, y\}}\left(M_{N}^{\prime}\right)=\{x, y\}$. By consistency in voters, for all $M_{N}^{\prime \prime} \in\left(2^{\mathcal{K}}\right)^{N}, v^{\{x, y\}}\left(M_{N}^{\prime \prime}\right)=\{x, y\}$. Thus, condition (10) holds if and only if $p_{x}^{\{x, y\}}=$ $p_{y}^{\{x, y\}}=0$. 
2. Suppose that $v^{\{x, y\}}\left(M_{N}\right)=\{x\}$ and $v^{\{x, y\}}\left(M_{N}^{\prime}\right)=\{x, y\}$. Assume $M_{N}^{\prime \prime}$ is such that $G_{x}\left(M_{N}^{\prime \prime}\right)=0$. Then, by consistency in voters (if $G_{y}\left(M_{N}^{\prime \prime}\right)>0$ ) or by the no-support condition (if $\left.G_{y}\left(M_{N}^{\prime \prime}\right)=0\right), v^{\{x, y\}}\left(M_{N}^{\prime \prime}\right)=\{x, y\}$. We observe that condition (10) holds for any $p_{x}^{\{x, y\}} \in \mathbb{R}_{++}$and $p_{y}^{\{x, y\}}=0$. Assume $M_{N}^{\prime \prime}$ is such that $G_{x}\left(M_{N}^{\prime \prime}\right)>$ 0. If $G_{y}\left(M_{N}^{\prime \prime}\right)=0$, the assumption $v^{\{x, y\}}\left(M_{N}\right)=\{x\}$ and consistency in voters imply that $v^{\{x, y\}}\left(M_{N}^{\prime \prime}\right)=\{x\}$. If $G_{y}\left(M_{N}^{\prime \prime}\right)>0$, define the response profiles $\hat{M}_{A}$ and $\hat{M}_{B}^{\prime}$ as $G_{y}\left(M_{N}^{\prime \prime}\right)$-isomorphic copies of $M_{N}^{\prime}$ and $G_{x}\left(M_{N}^{\prime \prime}\right)$-isomorphic copies of $M_{N}$, respectively. By consistency in voters, $v^{\{x, y\}}\left(\hat{M}_{A}\right)=\{x, y\}$ and $v^{\{x, y\}}\left(\hat{M}_{B}^{\prime}\right)=\{x\}$. Since $M_{N}^{\prime \prime}=\hat{M}_{A}+\hat{M}_{B}^{\prime}$, consistency in voters implies that $v^{\{x, y\}}\left(M_{N}^{\prime \prime}\right)=v^{\{x, y\}}\left(\hat{M}_{A}+\right.$ $\left.\hat{M}_{B}^{\prime}\right)=v^{\{x, y\}}\left(\hat{M}_{A}\right) \cap v^{\{x, y\}}\left(\hat{M}_{B}^{\prime}\right)=\{x\}$. Thus, condition (10) holds for any $p_{x}^{\{x, y\}} \in$ $\mathbb{R}_{++}$and $p_{y}^{\{x, y\}}=0$.

3. Suppose that $v^{\{x, y\}}\left(M_{N}\right)=\{x, y\}$ and $v^{\{x, y\}}\left(M_{N}^{\prime}\right)=\{y\}$. A symmetric argument as the one already used for the former case shows that condition (10) holds for $p_{x}^{\{x, y\}}=0$ and any $p_{y}^{\{x, y\}} \in \mathbb{R}_{++}$.

4. Suppose that $v^{\{x, y\}}\left(M_{N}\right)=\{x\}$ and $v^{\{x, y\}}\left(M_{N}^{\prime}\right)=\{y\}$. Then, condition (10) holds only if $p_{x}^{\{x, y\}}>0$ and $p_{y}^{\{x, y\}}>0$. To further restrict the weights in this case, we have to consider the response profiles in which both alternatives get at least one vote. Formally, define $\mathcal{M}_{N}=\left\{\bar{M}_{N} \in\left(2^{\mathcal{K}}\right)^{N}: G_{x}\left(\bar{M}_{N}\right)>0\right.$ and $\left.G_{y}\left(\bar{M}_{N}\right)>0\right\}$. We divide the analysis of this case into four subcases.

4.1 Assume that for all electorates $B$ and all $\tilde{M}_{B} \in \mathcal{M}_{B}, v^{\{x, y\}}\left(\tilde{M}_{B}\right)=\{x\}$. This contradicts coherence, and therefore, this case cannot be.

4.2 Assume that for all electorates $B$ and all $\tilde{M}_{B} \in \mathcal{M}_{B}, v^{\{x, y\}}\left(\tilde{M}_{B}\right)=\{y\}$. This contradicts coherence, and therefore, this case cannot be.

4.3 Assume that there exists an electorate $B$ and a response profile $\tilde{M}_{B} \in \mathcal{M}_{B}$ such that $v^{\{x, y\}}\left(\tilde{M}_{B}\right)=\{x, y\}$. Consider any electorate $A \neq B$ and any response profile $\hat{M}_{A} \in \mathcal{M}_{A}$. Assume at first that

$$
\frac{G_{x}\left(\hat{M}_{A}\right)}{G_{y}\left(\hat{M}_{A}\right)}>\frac{G_{x}\left(\tilde{M}_{B}\right)}{G_{y}\left(\tilde{M}_{B}\right)} .
$$


Since $x \in v^{\{x, y\}}\left(\tilde{M}_{B}\right)$ by assumption, Lemma 2 implies

$$
x \in v^{\{x, y\}}\left(\hat{M}_{A}\right) .
$$

Define the response profile $\bar{M}_{\bar{B}}$ as $G_{y}\left(\hat{M}_{A}\right)$-isomorphic copies of $\tilde{M}_{B}$. Since $v^{\{x, y\}}\left(\tilde{M}_{B}\right)=\{x, y\}$, consistency in voters implies $v^{\{x, y\}}\left(\bar{M}_{\bar{B}}\right)=\{x, y\}$. Define also the response profile $\bar{M}_{\bar{A}}^{\prime}$ as $G_{y}\left(\tilde{M}_{B}\right)$-isomorphic copies of $\hat{M}_{A}$. By (12), consistency in voters implies $x \in v^{\{x, y\}}\left(\bar{M}_{\bar{A}}^{\prime}\right)$. Observe that $G_{x}\left(\bar{M}_{\bar{A}}^{\prime}\right)=G_{y}\left(\tilde{M}_{B}\right)$. $G_{x}\left(\hat{M}_{A}\right), G_{x}\left(\bar{M}_{\bar{B}}\right)=G_{y}\left(\hat{M}_{A}\right) \cdot G_{x}\left(\tilde{M}_{B}\right)$, and $G_{y}\left(\bar{M}_{\bar{A}}^{\prime}\right)=G_{y}\left(\hat{M}_{A}\right) \cdot G_{y}\left(\tilde{M}_{B}\right)=$ $G_{y}\left(\bar{M}_{\bar{B}}\right)$. Let $t=G_{x}\left(\bar{M}_{\bar{A}}^{\prime}\right)-G_{x}\left(\bar{M}_{\bar{B}}\right)$. By $(11), t$ is a strictly positive integer. Let $\check{M}_{C}$ be a response profile obtained from $t$-isomorphic copies of $M_{N}$. Then, $\bar{M}_{\bar{A}}^{\prime}=\bar{M}_{\bar{B}}+\check{M}_{C}$. Since $v^{\{x, y\}}\left(M_{N}\right)=\{x\}$ by assumption and $x \in v^{\{x, y\}}\left(\bar{M}_{\bar{B}}\right)$, consistency in voters implies $v^{\{x, y\}}\left(\bar{M}_{\bar{A}}^{\prime}\right)=\{x\}$. Since $\frac{G_{x}\left(\bar{M}_{\bar{A}}^{\prime}\right)}{G_{y}\left(\bar{M}_{\bar{A}}^{\prime}\right)}=\frac{G_{y}\left(\tilde{M}_{B}\right) \cdot G_{x}\left(\hat{M}_{A}\right)}{G_{y}\left(\tilde{M}_{B}\right) \cdot G_{y}\left(\hat{M}_{A}\right)}=$ $\frac{G_{x}\left(\hat{M}_{A}\right)}{G_{y}\left(\hat{M}_{A}\right)}$, Lemma 1 implies finally that $v^{\{x, y\}}\left(\hat{M}_{A}\right)=v^{\{x, y\}}\left(\bar{M}_{\bar{A}}^{\prime}\right)=\{x\}$. Therefore,

$$
\text { if } \frac{G_{x}\left(\hat{M}_{A}\right)}{G_{y}\left(\hat{M}_{A}\right)}>\frac{G_{x}\left(\tilde{M}_{B}\right)}{G_{y}\left(\tilde{M}_{B}\right)} \text {, then } v^{\{x, y\}}\left(\hat{M}_{A}\right)=\{x\} \text {. }
$$

Assume now that $v^{\{x, y\}}\left(\hat{M}_{A}\right)=\{x\}$. Since $v^{\{x, y\}}\left(\tilde{M}_{B}\right)=\{x, y\}$, Lemma 1 implies that $\frac{G_{x}\left(\hat{M}_{A}\right)}{G_{y}\left(\hat{M}_{A}\right)} \neq \frac{G_{x}\left(\tilde{M}_{B}\right)}{G_{y}\left(\tilde{M}_{B}\right)}$. Assume at first that $\frac{G_{x}\left(\hat{M}_{A}\right)}{G_{y}\left(\hat{M}_{A}\right)}<\frac{G_{x}\left(\tilde{M}_{B}\right)}{G_{y}\left(\tilde{M}_{B}\right)}$. Then, since $y \in$ $v^{\{x, y\}}\left(\tilde{M}_{B}\right)$ by assumption, we obtain from Lemma 2 that $y \in v^{\{x, y\}}\left(\hat{M}_{A}\right)=\{x\}$. This is a contradiction, and therefore, $\frac{G_{x}\left(\hat{M}_{A}\right)}{G_{y}\left(\hat{M}_{A}\right)}>\frac{G_{x}\left(\tilde{M}_{B}\right)}{G_{y}\left(\tilde{M}_{B}\right)}$. Hence,

$$
\text { if } v^{\{x, y\}}\left(\hat{M}_{A}\right)=\{x\} \text {, then } \frac{G_{x}\left(\hat{M}_{A}\right)}{G_{y}\left(\hat{M}_{A}\right)}>\frac{G_{x}\left(\tilde{M}_{B}\right)}{G_{y}\left(\tilde{M}_{B}\right)} \text {. }
$$

We conclude from (13) and (14) that

$$
v^{\{x, y\}}\left(\hat{M}_{A}\right)=\{x\} \text { if and only if } \frac{G_{x}\left(\hat{M}_{A}\right)}{G_{y}\left(\hat{M}_{A}\right)}>\frac{G_{x}\left(\tilde{M}_{B}\right)}{G_{y}\left(\tilde{M}_{B}\right)} .
$$

Symmetrically, we can obtain that

$$
v^{\{x, y\}}\left(\hat{M}_{A}\right)=\{y\} \text { if and only if } \frac{G_{x}\left(\hat{M}_{A}\right)}{G_{y}\left(\hat{M}_{A}\right)}<\frac{G_{x}\left(\tilde{M}_{B}\right)}{G_{y}\left(\tilde{M}_{B}\right)} .
$$

It follows from (15) and (16) that condition (10) holds if and only if $p_{x}^{\{x, y\}}=$ $G_{y}\left(\tilde{M}_{B}\right)$ and $p_{y}^{\{x, y\}}=G_{x}\left(\tilde{M}_{B}\right)$. 
4.4 Assume that for all electorates $B$ and all $\tilde{M}_{B} \in \mathcal{M}_{B}, v^{\{x, y\}}\left(\tilde{M}_{B}\right) \neq\{x, y\}$ and neither case (4.1) nor case (4.2) holds. Consider the electorate $N$ and a response profile $\hat{M}_{N}$ with the property that $G_{x}\left(\hat{M}_{N}\right)=G_{y}\left(\hat{M}_{N}\right)=1$. Suppose without loss of generality that $v^{\{x, y\}}\left(\hat{M}_{N}\right)=\{x\}$. Moreover, set $p_{y}^{\{x, y\}} \equiv 1$. It remains to be shown that there exists a weight $p_{x}^{\{x, y\}} \in(1,+\infty)$ such that for all electorates $B$ and all response profiles $\tilde{M}_{B}$ such that $G_{y}\left(\tilde{M}_{B}\right)>G_{x}\left(\tilde{M}_{B}\right)$, (a) if $v^{\{x, y\}}\left(\tilde{M}_{B}\right)=\{x\}$, then $p_{x}^{\{x, y\}}>\frac{G_{y}\left(\tilde{M}_{B}\right)}{G_{x}\left(\tilde{M}_{B}\right)}$ and (b) if $v^{\{x, y\}}\left(\tilde{M}_{B}\right)=\{y\}$, then $p_{x}^{\{x, y\}}<\frac{G_{y}\left(\tilde{M}_{B}\right)}{G_{x}\left(\tilde{M}_{B}\right)}$. Suppose otherwise; that is, there is no such weight $p_{x}^{\{x, y\}}$. Then, there exists an electorate $A$ and two response profiles $M_{A}$ and $M_{A}^{\prime}$ such that $v^{\{x, y\}}\left(M_{A}\right)=\{x\}, v^{\{x, y\}}\left(M_{A}^{\prime}\right)=\{y\}$ and $\frac{G_{y}\left(M_{A}\right)}{G_{x}\left(M_{A}\right)}>\frac{G_{y}\left(M_{A}^{\prime}\right)}{G_{x}\left(M_{A}^{\prime}\right)}$. But, since $\frac{G_{y}\left(M_{A}\right)}{G_{x}\left(M_{A}\right)}>\frac{G_{y}\left(M_{A}^{\prime}\right)}{G_{x}\left(M_{A}^{\prime}\right)}$ and $v^{\{x, y\}}\left(M_{A}^{\prime}\right)=\{y\}$, it follows from Lemma 2 that $y \in$ $v^{\{x, y\}}\left(M_{A}\right)=\{x\}$. This is a contradiction.

We have shown that for any pair of alternatives $x, y \in \mathcal{K}$, there are two non-negative weights, $p_{x}^{\{x, y\}}$ and $p_{y}^{\{x, y\}}$, such that the subfamily of voting rules $v^{\{x, y\}}$ is the Weighted Approval Voting relative to these weights (observe that the proof of Lemma 3 is constructive apart from part 4.4; in the final Section, we explain how the weights can be obtained in this case). Hence, for every alternative $x \in \mathcal{K}$ there are $\kappa-1$ weights, one weight that can be applied in combination with each alternative $y \neq x$. In the next two Lemmata we show that it is possible to construct a single weight for every alternative.

Lemma 4 Assume that the family of voting rules $v$ is consistent in alternatives and voters, anonymous, coherent and satisfies the no-support condition. Given alternative $x \in \mathcal{K}$, if $v^{\{x, y\}}$ is the Weighted Approval Voting relative to $p^{\{x, y\}}=\left(0, p_{y}^{\{x, y\}}\right)$ for some $y \neq x$, then for all $z \in \mathcal{K} \backslash\{x, y\}, v^{\{x, z\}}$ is the Weighted Approval Voting relative to $p^{\{x, z\}}=\left(0, p_{z}^{\{x, z\}}\right)$.

Proof: Suppose otherwise; that is, $p^{\{x, y\}}=\left(0, p_{y}^{\{x, y\}}\right)$ and for some $z \in \mathcal{K} \backslash\{x, y\}$, $p_{x}^{\{x, z\}}>0$. Consider any electorate $N$ of size $n \geq 1$ and let the response profile $M_{N}$ be such that $G_{y}\left(M_{N}\right)=G_{z}\left(M_{N}\right)=0$ and $G_{x}\left(M_{N}\right)=1$. Since $p_{x}^{\{x, z\}}>0$ and $v^{\{x, z\}}$ is by Lemma 3 the Weighted Approval Voting relative to $p^{\{x, z\}}=\left(p_{x}^{\{x, z\}}, p_{z}^{\{x, z\}}\right), v^{\{x, z\}}\left(M_{N}\right)=\{x\}$. Thus, 
by consistency in alternatives, $z \notin v^{\{x, y, z\}}\left(M_{N}\right)$. Moreover, since by assumption $v^{\{x, y\}}$ is the Weighted Approval Voting relative to $p^{\{x, y\}}=\left(0, p_{y}^{\{x, y\}}\right), v^{\{x, y\}}\left(M_{N}\right)=\{x, y\}$. Thus, by consistency in alternatives, $v^{\{x, y, z\}}\left(M_{N}\right) \notin\{\{x\},\{y\}\}$. Observe that $v^{\{x, y, z\}}\left(M_{N}\right) \notin$ $\{\{x\},\{y\}\}$ and $z \notin v^{\{x, y, z\}}\left(M_{N}\right)$ imply that $v^{\{x, y, z\}}\left(M_{N}\right)=\{x, y\}$. By consistency in alternatives, $v^{\{y, z\}}\left(M_{N}\right)=v^{\{x, y, z\}}\left(M_{N}\right) \cap\{y, z\}=\{y\}$. By the no-support condition, $v^{\{y, z\}}\left(M_{N}\right)=\{y, z\}$, which is a contradiction.

Lemma 5 Assume that the family of voting rules $v$ is consistent in alternatives and voters, anonymous, coherent and satisfies the no-support condition. Then, there exists a $\kappa$-tuple of weights $\left(p_{z}\right)_{z \in \mathcal{K}} \in \mathbb{R}_{+}^{\kappa}$ such that for all alternatives $x, y \in \mathcal{K}, v^{\{x, y\}}$ is the Weighted Approval Voting relative to $p^{\{x, y\}}=\left(p_{x}, p_{y}\right)$.

Proof: The proof is done by induction on the set of feasible alternatives $K \subset \mathcal{K}$. Take any $K \subset \mathcal{K}$ of cardinality two. By Lemma 3 , there are two weights $\left(p_{x}^{K}\right)_{x \in K} \in \mathbb{R}_{+}^{2}$ such that $v^{K}$ is the Weighted Approval Voting relative to $p^{K}$.

InduCtion Hypothesis: Suppose that given the set of feasible alternatives $K \subset \mathcal{K}$ of cardinality $k \geq 3$, there exists a $k$-tuple of weights $\left(p_{x}^{K}\right)_{x \in K} \in \mathbb{R}_{+}^{k}$ such that for all $x, y \in K$, $v^{\{x, y\}}$ is the Weighted Approval Voting relative to $p^{\{x, y\}}=\left(p_{x}^{K}, p_{y}^{K}\right)$.

We have to prove that if the set of feasible alternatives is equal to $K \cup\{z\}, z \notin K$, then there exists a $k+1$-tuple of weights $\left(p_{x}^{K \cup\{z\}}\right)_{x \in K \cup\{z\}} \in \mathbb{R}_{+}^{k+1}$ such that for all $x, y \in K \cup\{z\}$, $v^{\{x, y\}}$ is the Weighted Approval Voting relative to $p^{\{x, y\}}=\left(p_{x}^{K \cup\{z\}}, p_{y}^{K \cup\{z\}}\right)$.

For all alternatives $x \in K$, let $p_{x}^{K \cup\{z\}}=p_{x}^{K}$. Then, for all $x, y \in K, v^{\{x, y\}}$ is the Weighted Approval Voting relative to $p^{\{x, y\}}=\left(p_{x}^{K \cup\{z\}}, p_{y}^{K \cup\{z\}}\right)$ by the induction hypothesis. It remains to determine the weight $p_{z}^{K \cup\{z\}}$. To do so, we divide our analysis in two cases, but first, by Lemma 3 , we know that for all $x \in K$, there exist two weights $p_{x}^{\{x, z\}}$ and $p_{z}^{\{x, z\}}$ such that $v^{\{x, z\}}$ is the Weighted Approval Voting relative to these weights.

1. Suppose that for all $x \in K, p_{x}^{\{x, z\}}=0$. By Lemma 4, for all $y \neq x, p_{x}^{\{x, y\}}=0$. Hence, $p_{x}^{K \cup\{z\}}=0$ for all $x \in K$, because if it was the case that for some $x \in K$, $p_{x}^{K \cup\{z\}}>0$, then there would be an alternative $y \in K \backslash\{x\}$ such that $v^{\{x, y\}}$ would 
not be the Weighted Approval Voting relative to $p_{x}^{K}$ and $p_{y}^{K}$. This would contradict the induction hypothesis. Now, if $p_{z}^{\{x, z\}}=0$ for some $x \in K$, then, by Lemma 4, $p_{z}^{\{y, z\}}=0$ for all $y \in K$. In this case, we set $p_{z}^{K \cup\{z\}}=0$. If, on the other hand, $p_{z}^{\{x, z\}}>0$ for some $x \in K$, then it follows from the contrapositive of Lemma 4 that $p_{z}^{\{y, z\}}>0$ for all $y \in K$. In this case, any $p_{z}^{K \cup\{z\}} \in \mathbb{R}_{++}$will have the property that for all $x \in K, v^{\{x, z\}}$ is the Weighted Approval Voting relative to $p^{\{x, z\}}=\left(0, p_{z}^{K \cup\{z\}}\right)$.

2. Suppose that for alternative $x \in K, p_{x}^{\{x, z\}}>0$. If $p_{z}^{\{x, z\}}=0$, then, by Lemma 4 , $p_{z}^{\{y, z\}}=0$ for all $y \in K$. In this case, set $p_{z}^{K \cup\{z\}}=0$. Therefore, suppose from now on that $p_{z}^{\{x, z\}}>0$. By Remark 1 , the weights $p_{x}^{\{x, z\}}$ and $p_{z}^{\{x, z\}}$ are determined up to proportional changes; that is, if we multiply both by $\lambda>0$, then the result of the election does not change. Set $\lambda$ equal to $p_{x}^{K \cup\{z\}}=\lambda \cdot p_{x}^{\{x, z\}}$, or, $\lambda=p_{x}^{K \cup\{z\}} / p_{x}^{\{x, z\}}$ (note that by the contrapositive of Lemma $4, p_{x}^{\{x, z\}}>0$ implies that for all $y \neq x, p_{x}^{\{x, y\}}>0$; hence, by the induction hypothesis, the weight $p_{x}^{K}=p_{x}^{K \cup\{z\}}>0$ ). Similarly, let $p_{z}^{K \cup\{z\}}=\lambda \cdot p_{z}^{\{x, z\}}=p_{z}^{\{x, z\}} \cdot p_{x}^{K \cup\{z\}} / p_{x}^{\{x, z\}}$. Without loss of generality we can also define $p_{x}^{K \cup\{z\}} \equiv 1$. Then, $p_{z}^{K \cup\{z\}}=p_{z}^{\{x, z\}} / p_{x}^{\{x, z\}}$. Since $v^{\{x, z\}}$ is the Weighted Approval Voting relative to $\left(p_{x}^{\{x, z\}}, p_{z}^{\{x, z\}}\right)$ by Lemma 3 , we conclude that this subfamily is also the Weighted Approval Voting relative to $p^{\{x, z\}}=\left(p_{x}^{K \cup\{z\}}, p_{z}^{K \cup\{z\}}\right)$.

It remains to be shown that given alternative $y \in K \backslash\{x\}, v^{\{y, z\}}$ is the Weighted Approval Voting relative to $p^{\{y, z\}}=\left(p_{y}^{K \cup\{z\}}, p_{z}^{K \cup\{z\}}\right)$. To do so, we prove that there exists a $\mu>0$ such that $p_{y}^{K \cup\{z\}}=\mu \cdot p_{y}^{\{y, z\}}$ and $p_{z}^{K \cup\{z\}}=\mu \cdot p_{z}^{\{y, z\}}$. Rewrite the equations as $p_{y}^{K \cup\{z\}} \cdot p_{z}^{\{y, z\}}=p_{z}^{K \cup\{z\}} \cdot p_{y}^{\{y, z\}}$ (observe that the assumption $p_{z}^{\{x, z\}}>0$ implies that $p_{z}^{\{y, z\}}>0$ by the contrapositive of Lemma 4) and suppose otherwise. That is,

$$
\delta \equiv p_{z}^{K \cup\{z\}} \cdot p_{y}^{\{y, z\}}-p_{y}^{K \cup\{z\}} \cdot p_{z}^{\{y, z\}}>0 .
$$

Note that we can deal with the case $\delta<0$ using a symmetric argument. Let $\bar{p}_{z} \equiv \frac{n_{z}}{m_{z}}$ and $\bar{p}_{y} \equiv \frac{n_{y}}{m_{y}}$ be two rational numbers such that $\bar{p}_{z}<p_{z}^{K \cup\{z\}}, \bar{p}_{y}>p_{y}^{K \cup\{z\}}$, and

$$
\bar{p}_{z} \cdot p_{y}^{\{y, z\}}-\bar{p}_{y} \cdot p_{z}^{\{y, z\}}>0 \text {. }
$$

Here, $n_{y}, m_{y}, n_{z}$ and $m_{z}$ are strictly positive integers. Observe that $\bar{p}_{y}$ and $\bar{p}_{z}$ must exist, because the set of rational numbers is dense in the set of real numbers. Rewrite 
equation (17) as

$$
p_{y}^{\{y, z\}} \cdot\left(n_{z} \cdot m_{y}\right)>p_{z}^{\{y, z\}} \cdot\left(n_{y} \cdot m_{z}\right) .
$$

Consider now the electorate $N$ of size $n \geq \min \left\{n_{y} \cdot n_{z}, n_{y} \cdot m_{z}, n_{z} \cdot m_{y}\right\}$ and let the response profile $M_{N}$ be such that $G_{x}\left(M_{N}\right)=n_{z} \cdot n_{y}, G_{y}\left(M_{N}\right)=n_{z} \cdot m_{y}$, and $G_{z}\left(M_{N}\right)=n_{y} \cdot m_{z}$. Since, by Lemma $3, v^{\{y, z\}}$ is the Weighted Approval Voting relative to $p^{\{y, z\}}=\left(p_{y}^{\{y, z\}}, p_{z}^{\{y, z\}}\right), v^{\{y, z\}}\left(M_{N}\right)=\{y\}$ by equation (18). This implies, by consistency in alternatives, that $z \notin v^{\{x, y, z\}}\left(M_{N}\right)$.

In addition, $G_{x}\left(M_{N}\right)=\bar{p}_{y} \cdot G_{y}\left(M_{N}\right)>p_{y}^{K \cup\{z\}} \cdot G_{y}\left(M_{N}\right)$. Since $v^{\{x, y\}}$ is the Weighted Approval Voting relative to $p^{\{x, y\}}=\left(1, p_{y}^{K \cup\{z\}}\right)$ by construction, $v^{\{x, y\}}\left(M_{N}\right)=\{x\}$. This implies, by consistency in alternatives, that $y \notin v^{\{x, y, z\}}\left(M_{N}\right)$.

The two conditions $z \notin v^{\{x, y, z\}}\left(M_{N}\right)$ and $y \notin v^{\{x, y, z\}}\left(M_{N}\right)$ imply together that $v^{\{x, y, z\}}\left(M_{N}\right)=\{x\}$. Hence, $v^{\{x, y, z\}}\left(M_{N}\right) \cap\{x, z\}=\{x\}$ and, by consistency in alternatives, $v^{\{x, z\}}\left(M_{N}\right)=\{x\}$. Finally, since $v^{\{x, z\}}$ is the Weighted Approval Voting relative to $p^{\{x, z\}}=\left(1, p_{z}^{K \cup\{z\}}\right)$ by construction, $v^{\{x, z\}}\left(M_{N}\right)=\{x\}$ is equivalent to $p_{z}^{K \cup\{z\}} \cdot G_{z}\left(M_{N}\right)<1 \cdot G_{x}\left(M_{N}\right)$. But $p_{z}^{K \cup\{z\}} \cdot G_{z}\left(M_{N}\right)>\bar{p}_{z} \cdot G_{z}\left(M_{N}\right)=\frac{n_{z}}{m_{z}} \cdot n_{y} \cdot m_{z}=$ $G_{x}\left(M_{N}\right)$. This is a contradiction.

Hence, there is a $(k+1)$-tuple of non-negative and finite weights $\left(p_{x}^{K \cup\{z\}}\right)_{x \in K \cup\{z\}}$ such that for all $x, y \in K \cup\{z\}, v^{\{x, y\}}$ is the Weighted Approval Voting relative to $p^{\{x, y\}}=$ $\left(p_{x}^{K \cup\{z\}}, p_{x}^{K \cup\{z\}}\right)$. The Lemma follows finally from the case $K \cup\{z\}=\mathcal{K}$ and $p_{x} \equiv p_{x}^{\mathcal{K}}$ for all $x \in \mathcal{K}$.

In the last step of the proof, we apply consistency in alternatives to generalize Lemma 5 to all sets of feasible alternatives.

Proof of Theorem 1: Any Weighted Approval Voting satisfies consistency in alternatives and voters, anonymity, the no-support condition and coherence. To prove the other implication let $v$ be a family of voting rules that satisfies consistency in alternatives and voters, anonymity, the no-support condition, and coherence. We show that the $\kappa$-tuple of non-negative and finite weights $\left(p_{x}\right)_{x \in \mathcal{K}}$ identified in Lemma 5 is such that for all sets of feasible alternatives $K$, all profiles $M$, and all electorates $N$, 
$x \in v^{K}\left(M_{N}\right)$ if and only if $p_{x} \cdot G_{x}\left(M_{N}\right) \geq p_{y} \cdot G_{y}\left(M_{N}\right)$ for all $y \in K$.

Assume that $x \in v^{K}\left(M_{N}\right)$. Then, by consistency in alternatives, $x \in v^{\{x, y\}}\left(M_{N}\right)$ for all $y \in K \backslash\{x\}$. By Lemma $5, v^{\{x, y\}}$ is the Weighted Approval Voting relative to $p^{\{x, y\}}=\left(p_{x}, p_{y}\right)$. Hence, $p_{x} \cdot G_{x}\left(M_{N}\right) \geq p_{y} \cdot G_{y}\left(M_{N}\right)$ for all $y \in K$.

Assume that $p_{x} \cdot G_{x}\left(M_{N}\right) \geq p_{y} \cdot G_{y}\left(M_{N}\right)$ for all $y \in K$. Then, for all $y \neq x, x \in$ $v^{\{x, y\}}\left(M_{N}\right)$ because, by Lemma $5, v^{\{x, y\}}$ is the Weighted Approval Voting relative to $p^{\{x, y\}}=$ $\left(p_{x}, p_{y}\right)$. If there is some $z \neq x$ such that $z \in v^{K}\left(M_{N}\right)$, then the set $v^{K}\left(M_{N}\right) \cap\{x, z\} \neq \emptyset$. Hence, $v^{\{x, z\}}\left(M_{N}\right)=v^{K}\left(M_{N}\right) \cap\{x, z\}$ by consistency in alternatives. Since, by Lemma 5 , $v^{\{x, z\}}$ is the Weighted Approval Voting relative to $p^{\{x, z\}}=\left(p_{x}, p_{z}\right), x \in v^{\{x, z\}}\left(M_{N}\right)$. Hence, $x \in v^{K}\left(M_{N}\right)$. If there does not exist any alternative $z \neq x$ such that $z \in v^{K}\left(M_{N}\right)$, then $x \in v^{K}\left(M_{N}\right)$ because the set $v^{K}\left(M_{N}\right)$ cannot be empty.

\section{$5 \quad$ Final Remarks}

We show next, with the help of five examples, the independence of the properties used

in Theorem 1. Afterwards, we indicate a procedure to obtain the weights in case 4.4 of Lemma 3, the only subcase where our proof is not constructive. We finish the paper with two remarks. We show in Subsection 5.3 how to strengthen the no-support condition to obtain Weighted Approval Voting with strictly positive weights and describe in Subsection 5.4 some similarities between the construction of our proof and proofs used in the literatures of Weighted Utilitarism and Ranking of Opportunity Sets.

\subsection{Independence of the Axioms}

Consistency in Alternatives: Fix $x \in \mathcal{K}$. Let the family of voting rules $v$ be such that for all sets of feasible alternatives $K$ of size two, all profiles $M$, and all electorates $N$, $v^{K}\left(M_{N}\right)=v_{A}^{K}\left(M_{N}\right)$. Otherwise, apply the Weighted Approval Voting with weights $p_{x}=2$ and $p_{y}=1$ for all $y \neq x$. This family satisfies consistency in voters, anonymity, the nosupport condition and coherence. The following example shows that it is not consistent 
in alternatives. Let $\mathcal{K}=\{x, y, z\}$ and suppose that $N=\{i, j\}$. If $M_{i}=M_{j}=\mathcal{K}$, then $v^{\{x, y\}}\left(M_{i}+M_{j}\right)=\{x, y\}$ and $v^{\{x, y, z\}}\left(M_{i}+M_{j}\right)=\{x\}$. Consistency in alternatives would imply that $v^{\{x, y\}}\left(M_{i}+M_{j}\right)=v^{\{x, y, z\}}\left(M_{i}+M_{j}\right) \cap\{x, y\}=\{x\}$. Hence, $v$ does not satisfy consistency in alternatives.

Consistency in Voters: Let the family of voting rules $v$ be such that for all sets of feasible alternatives $K$, all profiles $M$, and all electorates $N$, if $G_{x}\left(M_{N}\right)>1$ for some $x \in K$, then $v^{K}\left(M_{N}\right)=v_{A}^{K}\left(M_{N}\right)$. Otherwise, apply the Weighted Approval Voting with weights $p_{x}=2$ and $p_{y}=1$ for all $y \neq x$. This family satisfies consistency in alternatives, anonymity, the no-support condition, and coherence. The following example shows that it is not consistent in voters. Let $\mathcal{K}=\{x, y, z\}$ and suppose that $N=\{i, j\}$. If $M_{i}=M_{j}=\{x, y\}$, then $v^{\{x, y\}}\left(M_{i}\right)=v^{\{x, y\}}\left(M_{j}\right)=\{x\}$ and $v^{\{x, y\}}\left(M_{i}+M_{j}\right)=\{x, y\}$. Consistency in voters would imply that $v^{\{x, y\}}\left(M_{i}+M_{j}\right)=v^{\{x, y\}}\left(M_{i}\right) \cap v^{\{x, y\}}\left(M_{j}\right)=\{x\}$. Hence, $v$ does not satisfy consistency in voters.

Anonymity: Assign to each voter $i \in \mathbb{N}$ a strictly positive number $q_{i}$ in such a way that $q_{i} \neq q_{j}$ for some pair $i, j \in \mathbb{N}$. Now, let the family of voting rules $v$ be such that for all sets of feasible alternatives $K$, all profiles $M$, and all electorates $N, x \in v^{K}\left(M_{N}\right)$ if and only if $\sum_{i \in N: x \in M_{i}} q_{i} \geq \sum_{i \in N: y \in M_{i}} q_{i}$ for all $y \in K$. This family satisfies consistency in alternatives and voters, the no-support condition, and coherence. The following example shows that it is not anonymous. Let $\mathcal{K}=\{x, y, z\}$ and suppose that $N=\{i, j\}$. Moreover, let $q_{i}=2$ and $q_{j}=1$. If $M_{i}=M_{j}^{\prime}=\{x\}$ and $M_{i}^{\prime}=M_{j}=\{y\}$, then $v^{\{x, y\}}\left(M_{i}+M_{j}\right)=\{x\}$ and $v^{\{x, y\}}\left(M_{i}^{\prime}+M_{j}^{\prime}\right)=\{y\}$. Hence, $v$ does not satisfy anonymity.

No-Support: Let the family of voting rules $v$ be such that for all sets of feasible alternatives $K$, all profiles $M$, and all electorates $N, x \in v^{K}\left(M_{N}\right)$ if and only if $G_{x}\left(M_{N}\right) \leq G_{y}\left(M_{N}\right)$ for all $y \in K$. This family satisfies consistency in alternatives and voters, anonymity, and coherence. The following example shows that it does not satisfy the no-support condition. Let $\mathcal{K}=\{x, y, z\}$ and suppose that $N=\{i, j\}$. If $M_{i}=M_{j}=\{x\}$, then $v^{\{x, y\}}\left(M_{i}+M_{j}\right)=$ $\{y\}$. Hence, $v$ does not satisfy the no-support condition. 
Coherence: Let $\eta: \mathcal{K} \rightarrow\{1, \ldots, \kappa\}$ be any one-to-one mapping that assigns to every $x \in \mathcal{K}$ a natural number between 1 and $\kappa$. Given $\eta$, let the family of voting rules $v$ be such that for all sets of feasible alternatives $K$, all profiles $M$, and all electorates $N, v^{K}\left(M_{N}\right)=\{y \in$ $K: G_{y}\left(M_{N}\right)>0$ and $\eta(y)<\eta(z)$ for all $z \in K$ s.t. $\left.G_{z}\left(M_{N}\right)>0\right\}$. If no alternative gets any vote, then $v^{K}\left(M_{N}\right)=K$. This family satisfies consistency in alternatives and voters, anonymity, and the no-support condition. The following example shows that it does not satisfy coherence. Let $\mathcal{K}=\{x, y, z\}$ and define $\eta$ to be such that $\eta(x)=1$ and $\eta(y)=2$. Then, for all profiles $M$ and all electorates $N$ such that $G_{x}\left(M_{N}\right)>0$ and $G_{y}\left(M_{N}\right)>0$, $v^{\{x, y\}}\left(M_{N}\right)=\{x\}$. Hence, $v$ does not satisfy coherence.

An additional point which is also related to the independence of the properties regards the question whether it is possible to obtain a similar characterization of all Weighted Approval Voting for a given electorate (given set of feasible alternatives); that is, if the electorate (the set of feasible alternatives) is fixed at $N(K)$ and the corresponding consistency property is dropped, is the class of all Weighted Approval Voting characterized by the remaining four properties? The following two examples show that this is not the case.

Example 1: Suppose that the electorate is equal to $N=\{1,2\}$ and the set of alternatives is $\mathcal{K}=\{x, y, z\}$. Let the family of voting rules $\hat{v}=\left\{\hat{v}^{K, N}:\left(2^{\mathcal{K}}\right)^{N} \rightarrow 2^{K} \backslash\{\emptyset\}\right\}_{K}$ be such that for all sets of feasible alternatives $K \subseteq \mathcal{K}$ and all response profiles $M_{N} \in\left(2^{\mathcal{K}}\right)^{N}$ such that $G_{r}\left(M_{N}\right)>0$ for some $r \in \mathcal{K}, \hat{v}^{K}\left(M_{N}\right)=\left\{r \in K: G_{r}\left(M_{N}\right)>0\right\}$. If no alternatives receives any vote, the set $K$ is elected. This family of voting rules satisfies consistency in alternatives, anonymity, coherence, and the no-support condition. Yet, $\hat{v}$ is not a Weighted Approval Voting because there does not exist a vector of finite weights $p=\left(p_{x}, p_{y}, p_{z}\right) \in \mathbb{R}_{+}^{3}$ such that for all $K \subseteq \mathcal{K}$ and all response profiles $M_{N} \in\left(2^{\mathcal{K}}\right)^{N}$,

$$
r \in \hat{v}^{K}\left(M_{N}\right) \text { if and only if } p_{r} \cdot G_{r}\left(M_{N}\right) \geq p_{s} \cdot G_{s}\left(M_{N}\right) \text { for all } s \in K \text {. }
$$

To see it, consider the set of feasible alternatives $K=\{x, y\}$ and two response profiles $M_{N}$ and $M_{N}^{\prime}$ with the property that $G_{x}\left(M_{N}\right)=G_{y}\left(M_{N}^{\prime}\right)=1$ and $G_{x}\left(M_{N}^{\prime}\right)=G_{y}\left(M_{N}\right)=2$. Observe that, by definition of $\hat{v}, \hat{v}^{\{x, y\}}\left(M_{N}\right)=\hat{v}^{\{x, y\}}\left(M_{N}^{\prime}\right)=\{x, y\}$. Let $\left(p_{x}, p_{y}\right) \in \mathbb{R}_{+}^{2}$ be an arbitrary vector of finite and non-negative weights. Condition (19) implies simultaneously 
that $p_{x}=2 \cdot p_{y}$ and $2 \cdot p_{x}=p_{y}$. Hence, $p_{x}=p_{y}=0$. Now take any response profile $M_{N}^{\prime \prime}$ which satisfies $G_{x}\left(M_{N}^{\prime \prime}\right)>0$ and $G_{y}\left(M_{N}^{\prime \prime}\right)=0$. Since $\hat{v}\left(M_{N}^{\prime \prime}\right)=\{x\}$ by definition, condition (19) implies that $p_{x}>0$. This contradicts $p_{x}=0$.

Example 2: Suppose that the set of feasible alternatives is equal to $K=\{x, y, z\}$. Let the family of voting rules $\tilde{v}=\left\{\tilde{v}^{K, N}:\left(2^{K}\right)^{\mathbb{N}} \rightarrow 2^{K} \backslash\{\emptyset\}\right\}_{N}$ be such that for all profiles $M \in\left(2^{K}\right)^{\mathbb{N}}$ and all electorates $N$, (a) if $G_{x}\left(M_{N}\right)=G_{y}\left(M_{N}\right)>0$ and $G_{z}\left(M_{N}\right)=0$, $\tilde{v}^{K}\left(M_{N}\right)=\{x\},(\mathrm{b})$ if $G_{y}\left(M_{N}\right)=G_{z}\left(M_{N}\right)>0$ and $G_{x}\left(M_{N}\right)=0, \tilde{v}^{K}\left(M_{N}\right)=\{y\}$, and (c) if $G_{x}\left(M_{N}\right)=G_{z}\left(M_{N}\right)>0$ and $G_{y}\left(M_{N}\right)=0, \tilde{v}^{K}\left(M_{N}\right)=\{z\}$. Otherwise, $\tilde{v}^{K}\left(M_{N}\right)=$ $v_{A}^{K}\left(M_{N}\right)$. This family of voting rules is consistent in voters, anonymous (it depends only on the amount of votes every alternative receives), coherent (given an alternative, there is a situation in which all alternatives have strictly positive support and the considered alternative belongs to the image), and satisfies the no-support condition (if an alternative does not get any vote it is selected if and only if all alternatives have zero support). Yet, $\tilde{v}$ is not a Weighted Approval Voting because the cycle induces non-transitive weights for pairs of alternatives. To see it, assume otherwise, and let $p=\left(p_{x}, p_{y}, p_{z}\right) \in \mathbb{R}_{+}^{3}$ be the vector of weights associated to $\tilde{v}$. Then, (a) implies that $p_{x}>p_{y}$, (b) implies that $p_{y}>p_{z}$, and (c) implies that $p_{z}>p_{x}$, a contradiction.

\subsection{The Weights in Case 4.4 of Lemma 3}

Figure 1 below indicates the first steps of a procedure to obtain the weights $p_{x}^{\{x, y\}}$ and $p_{y}^{\{x, y\}}$ in the subcase 4.4 of Lemma 3, the situation when, for response profiles in which both alternatives receive strictly positive support, never $\{x, y\}$ is elected and neither $\{x\}$ nor $\{y\}$ are always elected. Since, in this case, both weights have to be strictly positive, we can normalize them. Without loss of generality, assume that the subfamily of voting rules $v^{\{x, y\}}$ has the property that for all response profiles $M_{N}$ with $G_{x}\left(M_{N}\right)=G_{y}\left(M_{N}\right)=1$,

$$
v^{\{x, y\}}\left(M_{N}\right)=\{x\} .
$$

Set $p_{y}^{\{x, y\}} \equiv 1$. To obtain $p_{x}^{\{x, y\}} \in \mathbb{R}_{++}$, we construct a tree that resembles all possible subfamilies $v^{\{x, y\}}$ (satisfying condition (20); otherwise, a symmetric argument to obtain 
$p_{y}^{\{x, y\}} \in \mathbb{R}_{++}$will follow with $\left.p_{x}^{\{x, y\}} \equiv 1\right)$. Namely, each subfamily $v^{\{x, y\}}$ satisfying condition (20) is identified with a particular branch in the tree and, vice versa, each branch in the tree can be identified with a unique subfamily $v^{\{x, y\}}$ satisfying condition (20). A node in this tree corresponds to sizes of electorates. Then, we assign to the $t^{\text {th }}$-node of a particular branch (which corresponds to any electorate of at most $t$ voters) a triple $\left(G_{t}, z_{t}, P_{t}\right)$. In this triple, $G_{t}=\left(G_{x}^{t}, G_{y}^{t}\right)$ corresponds to any response profile $M_{N}$, where $G_{x}\left(M_{N}\right)=G_{x}^{t}$ and $G_{y}\left(M_{N}\right)=G_{y}^{t}$. Given $G_{t}=\left(G_{x}^{t}, G_{y}^{t}\right)$, any response profile $M_{N}$ with this property will be called decisive, because it restricts the weight of $x$ for electorates of size $t$. Moreover, $z_{t}=v^{\{x, y\}}\left(M_{N}\right)$ is equal to the alternative elected at a decisive response profile $G_{t}$ (remember, $\{x, y\}$ is never elected) and $P_{t}=\left(l_{t}, u_{t}\right), 0<l_{t} \leq u_{t}$ with $u_{t} \in \mathbb{R}_{++} \cup\{+\infty\}$, is the set of weights from which $p_{x}^{\{x, y\}}$ could still be selected. As we will argue later on, the final weight assigned to $x$ is the (irrational) number $p_{x}^{\{x, y\}}$ such that $\lim _{t \rightarrow \infty} l_{t}=p_{x}^{\{x, y\}}=\lim _{t \rightarrow \infty} u_{t}$.

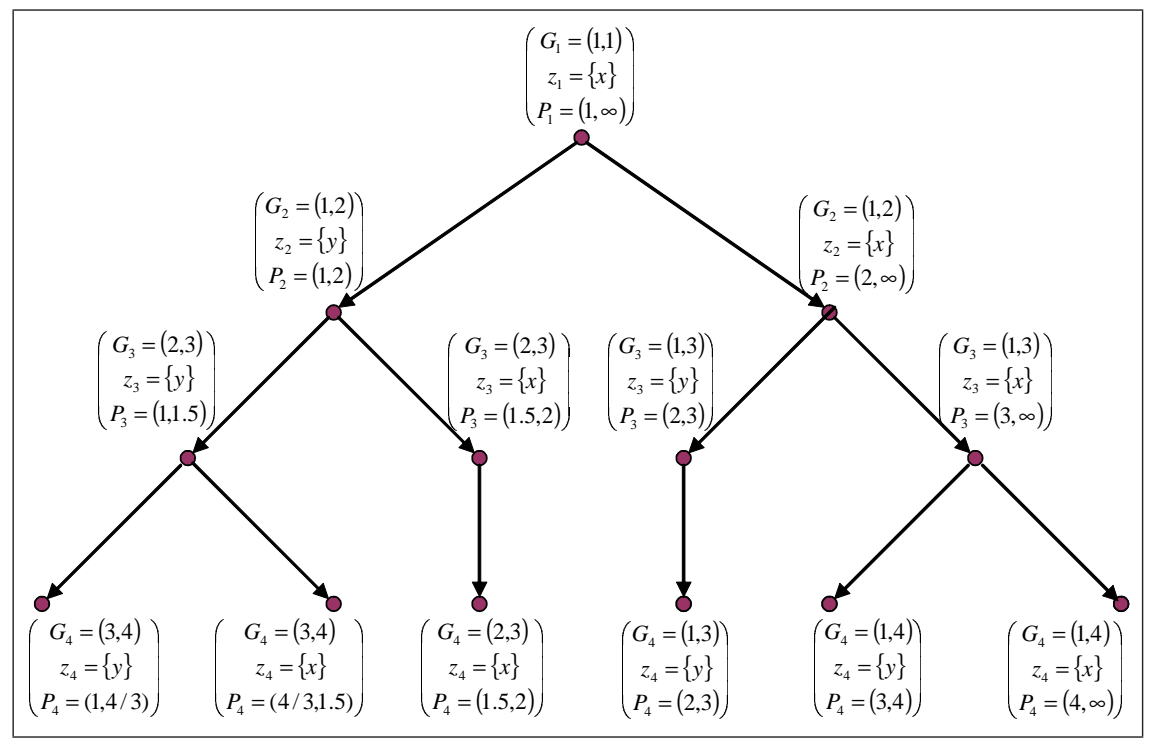

Figure 1: Construction of Weights

$t=1$. Any decisive response profile $M_{N}$ is such that $G_{x}\left(M_{N}\right)=G_{y}\left(M_{N}\right)=1$. We have assumed, without loss of generality, that $v^{\{x, y\}}\left(M_{N}\right)=\{x\}$. Then, $z_{1}=\{x\}$. Since $p_{y}^{\{x, y\}}=1$, we obtain $P_{1}=(1,+\infty)$ because any $p_{x}^{\{x, y\}} \in(1,+\infty)$ could still be selected. This case corresponds to the top node in Figure 1. 
$t=2$. Observe first that, by Lemma 2 , at every response profile $M_{N}$ such that $G_{x}\left(M_{N}\right) \geq$ $G_{y}\left(M_{N}\right), v^{\{x, y\}}\left(M_{N}\right)=\{x\}$. Thus, we restrict our attention to response profiles $M_{N}$ with the property that $G_{x}\left(M_{N}\right)<G_{y}\left(M_{N}\right)$. Hence, any response profile $M_{N}^{\prime}$ where $G_{x}\left(M_{N}^{\prime}\right)=1$ and $G_{y}\left(M_{N}^{\prime}\right)=2$ is decisive. If $v^{\{x, y\}}\left(M_{N}^{\prime}\right)=\{x\}$, then set $P_{2}=$ $(2,+\infty)$ because all weights below 2 have to be excluded and any $p_{x}^{\{x, y\}} \in(2,+\infty)$ could still be selected. On the other hand, if $v^{\{x, y\}}\left(M_{N}^{\prime}\right)=\{y\}$, then set $P_{2} \in(1,2)$ because all weights above 2 have to be excluded and any $p_{x}^{\{x, y\}} \in(1,2)$ could still be selected. These two cases correspond to either node $\left(G_{2}=(1,2), z_{2}=\{x\}, P_{2}=\right.$ $(2,+\infty))$ or node $\left(G_{2}=(1,2), z_{2}=\{y\}, P_{2}=(1,2)\right)$ in Figure 1.

$t=3$. If $\{x\}$ has been elected at the decisive response profile for $t=2$ (the former case above), then any decisive response profile $M_{N}$ for $t=3$ is such that $G_{x}\left(M_{N}\right)=1$ and $G_{y}\left(M_{N}\right)=3$. If $v^{\{x, y\}}\left(M_{N}\right)=\{x\}$ then set $P_{3}=(3,+\infty)$ and if $v^{\{x, y\}}\left(M_{N}\right)=\{y\}$ then set $P_{3}=(2,3)$. On the other hand, if $\{y\}$ has been elected instead (the latter case above), then any response profile $M_{N}$, where $G_{x}\left(M_{N}\right)=2$ and $G_{y}\left(M_{N}\right)=3$, becomes decisive for $t=3$. If $v^{\{x, y\}}\left(M_{N}\right)=\{x\}$ then set $P_{3}=(1.5,2)$ and if $v^{\{x, y\}}\left(M_{N}\right)=\{y\}$ then set $P_{3}=(1,1.5)$.

Observe that for some $t$ 's (for instance, for $t=4$ ), there exist subfamilies of voting rules $v^{\{x, y\}}$ with the property that their images are fully determined by the analysis of smaller electorates and Lemma 2. In these cases, the set of possible weights is not adjusted. We conclude our description by presenting a recursive algorithm that calculates, for a given $v^{\{x, y\}}$, the weight $p_{x}^{\{x, y\}}$. Suppose that the triple $\left(G_{t}, z_{t}, P_{t}\right)$ has already been calculated. Solve the program: Given $\left(G_{t}, z_{t}, P_{t}\right)$ choose $\left(G_{x}^{t+1}, G_{y}^{t+1}\right)$ to

$$
\begin{array}{ll}
\min G_{x}^{t+1}+G_{y}^{t+1} \text { s.t. } & \text { if } z_{t}=\{x\} \Rightarrow t+1 \geq G_{y}^{t+1}>G_{y}^{t} \text { and } t+1 \geq G_{x}^{t+1} \geq G_{x}^{t}, \\
& \text { if } z_{t}=\{y\} \Rightarrow t+1 \geq G_{y}^{t+1} \geq G_{y}^{t} \text { and } t+1 \geq G_{x}^{t+1}>G_{x}^{t}, \\
& G_{x}^{t+1}<G_{y}^{t+1}, \text { and } \\
& \frac{G_{y}^{t+1}}{G_{x}^{t+1}} \in P_{t} .
\end{array}
$$

If this program has a unique solution, then any response profile $M_{N}$ with $G_{x}\left(M_{N}\right)=$ $G_{x}^{t+1}$ and $G_{y}\left(M_{N}\right)=G_{y}^{t+1}$ is decisive for $t+1$. Now, update the set $P_{t}$ as follows: If 
$v^{\{x, y\}}\left(M_{N}\right)=\{x\}$, then $l_{t+1}=\frac{G_{y}^{t+1}}{G_{x}^{t+1}}$ and $u_{t+1}=u_{t}$. Similarly, if $v^{\{x, y\}}\left(M_{N}\right)=\{y\}$, then

$l_{t+1}=l_{t}$ and $u_{t+1}=\frac{G_{y}^{t+1}}{G_{x}^{t+1}}$. Set $P_{t+1}=\left(l_{t+1}, u_{t+1}\right)$. Moreover, let $z_{t+1}=v^{\{x, y\}}\left(M_{N}\right)$. On the other hand, if this program does not have a solution, then set $\left(G_{t+1}, z_{t+1}, P_{t+1}\right)=$ $\left(G_{t}, z_{t}, P_{t}\right)$. This iterative process yields, for a given $v^{\{x, y\}}$, two sequences, $\left\{l_{t}\right\}_{t=1}^{\infty}$ and $\left\{u_{t}\right\}_{t=1}^{\infty}$. First, and since $v^{\{x, y\}}$ is such that there exists a response profile $M_{N} \in \mathcal{M}_{N}$ at which $v^{\{x, y\}}\left(M_{N}\right) \neq\{x\}$ (i.e., we are not in case 4.1 of Lemma 3), there must exist a $T>1$ with the property that $u_{T}=T$ and $l_{T}=T-1$. Moreover, $\left\{u_{t}\right\}_{t=T}^{\infty}$ is a decreasing sequence in the compact set $[T-1, T],\left\{l_{t}\right\}_{t=T}^{\infty}$ is increasing in $[T-1, T]$, and for all $t \geq 1, l_{t}<u_{t}$. Hence, both sequences converge and $\lim _{t \rightarrow \infty} l_{t} \equiv l \leq u \equiv \lim _{t \rightarrow \infty} u_{t}$. To see that $l=u$ suppose otherwise; that is, $u>l$. Then, there is a rational number $q \equiv \frac{n}{m} \in(l, u)$, because the rational numbers are dense in the set of real numbers. Take now any response profile $M_{N}$ which is such that $G_{x}\left(M_{N}\right)=m$ and $G_{y}\left(M_{N}\right)=n$. In this case, $q \cdot G_{x}\left(M_{N}\right)=G_{y}\left(M_{N}\right)$. Since the subfamily $v^{\{x, y\}}$ does never elect the set $\{x, y\}$, $v^{\{x, y\}}$ cannot be a Weighted Approval Voting relative to $p^{\{x, y\}}=(q, 1)$. Hence, $q$ cannot belong to the set $(l, u)$, and therefore, $u=l$. Observe finally that $p_{x}^{\{x, y\}}=u=l$ must be an irrational number, because otherwise there would again be a response profile for which the set $\{x, y\}$ is elected.

\subsection{No-Support Condition}

According to Theorem 1, the weights are non-negative. If we strengthen the no-support condition, then we obtain a similar result in which the weights have to be strictly positive.

Strong No-Support: The family of voting rules $v$ satisfies the strong no-support condition if for all $x, y \in \mathcal{K}$, all profiles $M, M^{\prime} \in\left(2^{K}\right)^{\mathbb{N}}$, and all electorates $N$ such that $\begin{aligned} G_{x}\left(M_{N}\right)=G_{y}\left(M_{N}\right)= & G_{x}\left(M_{N}^{\prime}\right)=0 \text { and } G_{y}\left(M_{N}^{\prime}\right)>0, \\ & v^{\{x, y\}}\left(M_{N}\right)=\{x, y\} \text { and } v^{\{x, y\}}\left(M_{N}^{\prime}\right)=\{y\} .\end{aligned}$

Corollary 1 The family of voting rules $v$ is consistent in alternatives and voters, anonymous, coherent and satisfies the strong no-support condition if and only if $v$ is a Weighted Approval Voting relative to $\left(p_{x}\right)_{x \in \mathcal{K}}$, where $p_{x} \in \mathbb{R}_{++}$for all $x \in \mathcal{K}$. 
If we now add neutrality to these properties, then we obtain Approval Voting. Formally, given a one-to-one mapping $\mu: \mathcal{K} \rightarrow \mathcal{K}$, called a permutation $\mu$ of $\mathcal{K}$, and given a response profile $M_{N}$, let $\mu\left(M_{N}\right)$ and $\mu(K)$ be the response profile and the set of alternatives obtained by permuting alternatives according to $\mu$.

Neutrality: The family of voting rules $v$ is neutral if for all $x, y \in \mathcal{K}$, all profiles $M$, all electorates $N$, and all permutations $\mu$ of $\mathcal{K}$,

$$
v^{\mu(\{x, y\})}\left(\mu\left(M_{N}\right)\right)=\mu\left(v^{\{x, y\}}\left(M_{N}\right)\right) .
$$

Corollary 2 The family of voting rules $v$ is consistent in alternatives and voters, anonymous, neutral, coherent, and satisfies the strong no-support condition if and only if $v$ is Approval Voting.

Observe that Corollary 2 is not tight, because there is no $v$ that violates coherence but satisfies consistency in alternatives and voters, anonymity, neutrality and the strong no-support condition.

\subsection{Weighted Utilitarianism and Ranking of Opportunity Sets}

Our proof uses a construction that has some similarities with arguments already used in the weighted utility theory as well as in the literature on the ranking of opportunity sets. In the first case (see Bossert and Weymark [6]), consider an individual with utility $u: \mathbb{R}_{+}^{2} \rightarrow \mathbb{R}$ over consumption bundles of two goods. Under a set of axioms it is shown that the utility function $u$ is weighted utilitarian; i.e., there exist two weights $q_{1} \geq 0$ and $q_{2} \geq 0$ such that for all $\left(x_{1}, x_{2}\right),\left(y_{1}, y_{2}\right) \in \mathbb{R}_{+}^{2}, u\left(x_{1}, x_{2}\right)>u\left(y_{1}, y_{2}\right)$ if and only if $q_{1} x_{1}+q_{2} x_{2}>q_{1} y_{1}+q_{2} y_{2}$. Then, using a separability condition, the utility function is extended from the two-goods case to the $n$-goods case. In the second literature, the aim is to order the family of all non-empty subsets of a given set of alternatives according to two principles related to the opportunities they convey: indirect utility (maximal element) and freedom of choice (cardinality of the set). Dutta and Sen [12] and Alcalde-Unzu and Ballester [1] show that generalized utilitarian rules (additive aggregation of the two criteria) are the unique ones 
that satisfy a desirable set of axioms. The construction is also based on identifying the two weights for every pair of subsets and then extend them in a consistent manner to the full power set.

\section{References}

[1] J. Alcalde-Unzu and M. Ballester, Some Remarks on Ranking Opportunity Sets and Arrow Impossibility Theorems: Correspondence Results, Journal of Economic Theory 124 (2005), 116-123.

[2] C. Alós-Ferrer, A Simple Characterization of Approval Voting, forthcoming Social Choice and Welfare.

[3] K. Arrow, Rational Choice Functions and Orderings, Economica 26 (1959), 121-127.

[4] N. Baigent and Y. Xu, Independent Necessary and Sufficient Conditions for Approval Voting, Mathematical Social Sciences 21 (1991), 21-29.

[5] A. Bogomolnaia, H. Moulin, and R. Stong, Collective Choice under Dichotomous Preferences, Journal of Economic Theory 122 (2005), 165-184.

[6] W. Bossert and J. Weymark, Utiliy in Social Choice, in "Handbook of Utiliy Theory Vol. II" (eds. S. Barberá, P. Hammond, and C. Seidl), Kluwer Academic, Amsterdam, 2004.

[7] S. Brams and P. Fishburn, Approval Voting, American Political Science Review 72 (1978), 831-847.

[8] __ Going from Theory to Practice: The Mixed Success of Approval Voting, Social Choice and Welfare 25 (2005), 457-474.

[9] S. Brams and M. Sanver, Critical Strategies under Approval Voting: Who Gets Ruled In and Ruled Out, mimeo (2005). 
[10] F. De Sinopoli, B. Dutta, and J.F. Laslier, Approval Voting: Three Examples, mimeo (2005).

[11] A. Dellis and M. Oak, Approval Voting with Endogenous Candidates, Games and Economic Behavior 54 (2006), 47-76.

[12] B. Dutta and A. Sen, Ranking Opportunity Sets and Arrow Impossibility Theorems: Correspondence Results, Journal of Economic Theory 74 (1996), 90-101.

[13] P. Fishburn, Symmetric and Consistent Aggregation with Dichotomous Preferences, in "Aggregation and Revelation of Preferences" (ed. J. Laffont), North-Holland, Amsterdam, 1978.

[14] _ Axioms for Approval Voting: Direct Proof, Journal of Economic Theory 19 (1978), 180-185.

[15] R Goodin and C. List, A Conditional Defense of Plurality Rule: Generalizing May's Theorem in a Restricted Informational Environment, forthcoming American Journal of Political Science.

[16] J.F. Laslier, Analyzing a Preference and Approval Profile, Social Choice and Welfare 20 (2003), 229-242.

[17] _ Spatial Approval Voting, Political Analysis 14 (2006), 160-185.

[18] S. Nitzan and E. Baharad, Approval Voting Reconsidered, Economic Theory 26 (2006), 619-628.

[19] M. Regenwetter and I. Tsetlin, Approval Voting and Positional Voting Methods: Inference, Relationship, Examples, Social Choice and Welfare 22 (2004), 539-566.

[20] M. Sertel, Characterizing Approval Voting, Journal of Economic Theory 45 (1988), 207-211. 
[21] H. Smith, Aggregation of Preferences with Variable Electorates, Econometrica 41 (1973), 1027-1041.

[22] M. Vorsatz, Approval Voting on Dichotomous Preferences, forthcoming Social Choice and Welfare.

[23] _ Scoring Rules on Dichotomous Preferences, mimeo (2005). 